\title{
DIFFERENTIAL SYSTEMS ON FIBERED MANIFOLDS
}

\section{A. M. RODRIGUES}

Let $M$ and $M^{\prime}$ be real analytic manifolds and $\rho: M \rightarrow M^{\prime}$ an analytic map which is surjective and whose rank is equal to the dimension of $M^{\prime}$ at every point of $M$. We shall denote respectively by $\rho_{*}$ and $\rho^{*}$ the maps induced by $\rho$ on tangent vectors and differential forms.

Let $\Sigma$ and $\Sigma^{\prime}$ be analy tic exterior differential systems defined respectively in $M$ and $M^{\prime}$ and assume that for every differential form $\omega \in \Sigma^{\prime}, \rho^{*} \omega$ belongs to $\Sigma$. Take a point $x_{0} \in M$ and put $x_{0}^{\prime}=\rho\left(x_{0}\right)$. Let $\mathcal{V}^{\prime p}$ be an integral manifold of $\Sigma^{\prime}$ going through $x_{0}^{\prime}$. In this note we give a condition for the existence of an integral manifold $v^{p}$ of $\Sigma$ going through $x_{0}$ such that, for a suitable neighborhood $U$ of $x_{0}$, $\rho\left(V^{p}\right)=V^{\prime p} \cap \rho(U)$.

The proof consists in a careful application to our situation of the technic of the Cartan-Kähler theory. The situation we study here appears in the theory of continuous pseudogroups (see [3, p. 125]). For the definitions and results we use of the theory of exterior differential systems we refer the reader to [1] and [2].

For any integral contact element $E^{k}\left(x_{0}\right)$ of $\Sigma$ denote by $J\left(E^{k}\left(x_{0}\right)\right)$ the polar space of $E^{k}\left(x_{0}\right)$ and by $J^{\prime}\left(E^{k}\left(x_{0}\right)\right)$ the subspace of $J\left(E^{k}\left(x_{0}\right)\right)$ of all forms $\omega\rfloor X_{1} \wedge \cdots \wedge X_{r}$, where $\omega$ is a form of degree $r+1$ belonging to $\rho^{*}\left(\Sigma^{\prime}\right)$ and $X_{1}, \cdots, X_{r}$ are vectors of $E^{k}\left(x_{0}\right)$.

Let $F_{x_{0}}$ be the tangent space to the fiber of $M$ at the point $x_{0}$ and denote by $J\left(E^{k}\left(x_{0}\right)\right) \mid F_{x_{0}}$ the space obtained restricting the forms of $J\left(E^{k}\left(x_{0}\right)\right)$ to the subspace $F_{x_{0}}$.

Denote by $E^{\prime p}\left(x_{0}^{\prime}\right)$ the tangent space of $\mathcal{V}^{\prime p}$ at $x_{0}^{\prime}$ and assume that there exists an ordinary integral element $E^{p}\left(x_{0}\right)$ of $\Sigma$ such that $\rho_{*}\left(E^{p}\left(x_{0}\right)\right)=E^{\prime p}\left(x_{0}\right)$. Assume moreover there exists a sequence $E^{0}\left(x_{0}\right)$ $\subset E^{1}\left(x_{0}\right) \subset \cdots \subset E^{p-1}\left(x_{0}\right)$ of regular contact elements contained in $E^{p}\left(x_{0}\right)$ and such that $\operatorname{dim} J\left(E^{k}\left(x_{0}\right)\right)-\operatorname{dim} J^{\prime}\left(E^{k}\left(x_{0}\right)\right)=$ $\operatorname{dim} J\left(E^{k}\left(x_{0}\right)\right) \mid F_{x_{0}}, 0 \leqq k \leqq p-1$. Under these assumptions we shall prove the following theorem.

TheOREm. There exists an integral manifold $v^{p}$ of $\Sigma$ defined in a neighborhood $U$ of $x_{0}$ such that the tangent space $\mathcal{V}_{x_{0}}^{p}$ of $\mho^{p}$ at the point $x_{0}$ is $E^{p}\left(x_{0}\right)$ and $\rho\left(\mathcal{V}^{p}\right)=\mathcal{V}^{\prime} p \rho(U)$.

Proof. Choose coordinates $x^{i}, 1 \leqq i \leqq n^{\prime}$ in $M^{\prime}$, defined in a neighborhood of $x_{0}^{\prime}$ and coordinates $x^{i}, y^{j}, 1 \leqq j \leqq n$ in $M$, defined in a

Received by the editors January 26, 1966. 
neighborhood of $x_{0}$ such that $d x^{k+1}\left|E^{k}\left(x_{0}\right)=\cdots=d x^{n}\right| E^{k}\left(x_{0}\right)$ $=d y^{j} \mid E^{k}\left(x_{0}\right)=0,1 \leqq k \leqq p, 1 \leqq j \leqq n$ (we shall use the same notation for $x^{i}$ and $\left.x^{i} \circ \rho\right)$. Since the differentials $d x^{1}, \cdots, d x^{p}$ are linearly independent on $E^{\prime p}\left(x_{0}\right), \mathcal{U}^{\prime p}$ is locally defined by equations $x^{\lambda}$ $=H^{\lambda}\left(x^{1}, \cdots, x^{p}\right), p+1 \leqq \lambda \leqq n^{\prime}$. Since $\mathcal{V}^{\prime p}$ is tangent to $E^{\prime p}\left(x_{0}^{\prime}\right)$ and $d x^{\lambda} \mid E^{\prime p}\left(x_{0}\right)=0,\left(\partial H^{\lambda} / \partial x^{i}\right)_{x 0}{ }^{\prime}=0$. Let $\mho^{\prime 1}$ be the curve in $M^{\prime}$ defined by equations $x^{2}=\cdots=x^{p}=0, x^{\lambda}=H^{\lambda}\left(x^{1}, 0, \cdots, 0\right), p+1 \leqq \lambda \leqq n^{\prime}$, and put $E^{\prime k}\left(x_{0}\right)=\rho_{*}\left(E^{k}\left(x_{0}\right)\right)$. Clearly $\mathcal{V}_{x_{0}}^{\prime 1}=E^{\prime 1}\left(x_{0}\right)$. We want to show that there is an in tegral curve of $\Sigma$ which covers $\mho^{\prime 1}$. Let $\bar{\eta}^{1}, \cdots, \bar{\eta}^{\alpha_{1}}$ be a basis of the space of forms of degree 1 of $\Sigma^{\prime}$ at the point $x_{0}^{\prime}$ and choose forms $\bar{\xi}^{1}, \cdots, \bar{\xi}^{\beta_{1}}$ such that $\bar{\eta}^{1}, \cdots, \bar{\eta}^{\alpha_{1}}, \bar{\xi}^{1}, \cdots, \bar{\xi}^{\beta_{1}}$ is a basis of the forms of degree 1 of $\Sigma$ at the point $x_{0}$ (we denote the form $\rho^{*} \bar{\eta}^{i}$ also by $\left.\bar{\eta}^{i}\right)$. Since $x_{0}$ is a regular point of $\Sigma$ there are forms $\eta^{1}, \cdots, \eta^{\alpha_{1}}, \xi^{1}, \cdots, \xi^{\beta_{1}}$ defined in a neighborhood of $x_{0}$ such that they are a basis of the space of 1 -forms of $\Sigma$ in this neighborhood and $\eta_{x_{0}}^{i}=\bar{\eta}^{i}, \xi_{x_{0}}^{i}=\bar{\xi}^{i}$. Put

$$
\begin{aligned}
\underset{\eta_{\beta}}{\alpha} & =\sum_{i=1}^{n^{\prime}} A_{i}^{\alpha}(x) d x^{i}, \quad 1 \leqq \alpha \leqq \alpha_{1}, \\
\xi^{\beta} & =\sum_{i=1}^{n^{\prime}} B_{i}^{\beta}(x, y) d x^{i}+\sum_{j=1}^{n} C_{j}^{\beta}(x, y) d y^{j}, \quad 1 \leqq \beta \leqq \beta_{1} .
\end{aligned}
$$

From the hypothesis, the matrix $\left\|C_{j}^{\beta}(x, y)\right\|$ has maximum rank at the point $x_{0}$. Hence, the linear equations

$$
B_{i}^{\beta}(x, y)+\sum_{\lambda=p+1}^{n^{\prime}} B_{\lambda}^{\beta} \frac{\partial H^{\lambda}}{\partial x^{1}}+\sum_{j=1}^{n} C_{j}^{\beta}(x, y) \frac{d y^{j}}{d x^{1}}=0
$$

can be solved with respect to some of the variables $d y^{j} / d x^{i}$. Assume that they can be solved with respect to $d y^{1} / d x^{1}, \cdots, d y^{s} / d x^{1}$. In (3), put, $x^{2}=\cdots=x^{n \prime}=0$ and replace the variables $y^{s+1}, \cdots, y^{n}$ by arbitrary functions $F^{s+1}\left(x^{1}\right), \cdots, F^{n}\left(x^{1}\right)$ such that $\left(d F^{k} / d x^{1}\right)=0$, $s+1 \leqq k \leqq n$. Let $F^{k}\left(x^{1}\right), 1 \leqq k \leqq s$, be the solution of the resulting system with the initial conditions $\left(d F^{k} / d x^{1}\right)_{x^{1}=0}=0,1 \leqq h \leqq s$. Then the curve $x^{2}=\cdots=x^{p}=0, x^{\lambda}=H^{\lambda}\left(x^{1}, 0, \cdots, 0\right), y^{j}=F^{j}\left(x^{1}\right), p+1 \leqq \lambda$ $\leqq n^{\prime}, 1 \leqq j \leqq n$, is an integral curve of $\Sigma$ which covers $\mathcal{V}^{\prime 1}$.

Assume now, by induction, that we have lifted the manifold $v^{\prime r-1}$ defined by the equations $x^{r}=\cdots=x^{p}=0, x^{\lambda}=H^{\lambda}\left(x^{1}, \cdots, x^{r-1}\right.$, $0, \cdots, 0)$ to an in tegral manifold $v^{r-1}$ of $\Sigma$ which is tangent $E^{r-1}\left(x_{0}\right)$. Let $\bar{\eta}^{1}, \cdots, \bar{\eta}^{\alpha_{r-1}}, \bar{\xi}^{1}, \cdots, \bar{\xi}^{\beta_{r-1}}$ be a basis of $J\left(E^{r-1}\left(x_{0}\right)\right)$ such that $\bar{\eta}^{1}, \cdots, \bar{\eta}^{\alpha_{r-1}}$, is a basis of $J^{\prime}\left(E^{r-1}\left(x_{0}\right)\right)$. For a contact element $E^{r}(x)$ sufficiently close to $E^{r}\left(x_{0}\right)$, we can write 


$$
\begin{array}{ll}
d x^{\lambda} \mid E^{r}(x)=\sum_{i=1}^{r} u_{i}^{\lambda} d x^{i}, & r+1 \leqq \lambda \leqq n^{\prime}, \\
d y^{\lambda} \mid E^{r}(x)=\sum_{i=1}^{r} v_{i}^{j} d x^{i}, & 1 \leqq j \leqq n .
\end{array}
$$

Put

$$
L_{i}\left(E^{r}(x)\right)=\frac{\partial}{\partial x^{i}}+\sum_{\lambda=r+1}^{n^{\prime}} u_{i}^{\lambda} \frac{\partial}{\partial x^{\lambda}}+\sum_{j=1}^{n} v_{i}^{j} \frac{\partial}{\partial y^{j}}, \quad 1 \leqq i \leqq r,
$$

and assume that

$$
\left.\bar{\eta}^{\alpha}=\left(L_{i_{1}}\left(E^{r}\left(x_{0}\right)\right) \wedge \cdots \wedge L_{i_{t-1}}\left(E^{r}\left(x_{0}\right)\right)\right)\right\rfloor \omega, \quad 1 \leqq \alpha \leqq \alpha_{r-1},
$$

where $\omega$ is a form of degree $t$ in $\rho^{*} \Sigma^{\prime}$. For $E^{r}(x)$ sufficiently close to $E^{r}\left(x_{0}\right)$ define

$$
\eta^{\alpha}\left(E^{r}(x)\right)=\left(L_{i_{1}}\left(E^{r}(x)\right) \wedge \cdots \wedge L_{i_{t-1}}\left(E^{r}(x)\right)\right) \_\omega .
$$

Define forms $\xi^{\beta}$ in a similar way. Let

$$
\begin{gathered}
\eta^{\alpha}\left(E^{r}(x)\right)=\sum_{i=1}^{n^{\prime}} A_{i}^{\alpha}(x, u) d x^{i}, \\
\xi^{\beta}\left(E^{r}(x)\right)=\sum_{i=1}^{n^{\prime}} B_{i}^{\beta}(x, y, u, v) d x^{i}+\sum_{j=1}^{n} C_{j}^{\beta}(x, y, u, v) d y^{j},
\end{gathered}
$$

be the expression of these forms in local coordinates. Observe that the coefficients are functions only of the variables $x, y, u_{i}^{\lambda}, v_{i}^{j}$ with $1 \leqq i \leqq r-1$.

By hypothesis the matrix $\left\|C_{j}^{\beta}\right\|$ has maximum rank at the point $E^{r}\left(x_{0}\right)$. Assume that the equations of $\mho^{r-1}$ are $x^{r}=\cdots=x^{p}=0$, $x^{\lambda}=H^{\lambda}\left(x^{1}, \cdots, x^{r-1}, 0, \cdots, 0\right), y^{j}=F^{j}\left(x^{1}, \cdots, x^{r}\right)$ and construct the functions

$$
\begin{aligned}
& \eta^{\alpha}\left(E^{r}(x)\right)\left(L_{r}\left(E^{r}(x)\right)\right)=A_{r}^{\alpha}+\sum_{\lambda=r+1}^{n^{\prime}} A_{\lambda}^{\alpha} u_{r}^{\lambda}, \\
& \xi^{\beta}\left(E^{r}(x)\right)\left(L_{r}(E(x))=B_{r}^{\beta}+\sum_{\lambda=r+1}^{n^{\prime}} B_{\lambda}^{\beta} u_{r}^{\lambda}+\sum_{j=1}^{n} C_{j}^{\beta} v_{r}^{j} .\right.
\end{aligned}
$$

Consider the following system of partial differential equations, obtained replacing in the above functions $u_{i}^{\lambda}$ by $\partial x^{\lambda} / \partial x^{i}$ and $v_{i}^{j}$ by $\partial y^{j} / \partial x^{i}$ : 


$$
\begin{gathered}
A_{r}^{\alpha}\left(x, \frac{\partial x^{\lambda}}{\partial x^{i}}\right)+\sum_{\lambda=r+1}^{n^{\prime}} A_{\lambda}^{\alpha}\left(x, \frac{\partial x^{\lambda}}{\partial x^{i}}\right) \frac{\partial x^{\lambda}}{\partial x^{r}}=0, \\
B_{r}^{\beta}\left(x, y, \frac{\partial x^{\lambda}}{\partial x^{i}}, \frac{y^{j}}{\partial x^{i}}\right)+\sum_{\lambda=r+1}^{n^{\prime}} B_{\lambda}^{\beta}\left(x, y, \frac{\partial x^{\lambda}}{\partial x^{i}}, \frac{\partial y^{j}}{\partial x^{i}}\right) \frac{\partial x^{\lambda}}{\partial x^{r}} \\
+\sum_{j=1}^{n} C_{j}^{\beta}\left(x, y, \frac{\partial x^{\lambda}}{\partial x^{i}}, \frac{\partial y^{j}}{\partial x^{i}}\right) \frac{\partial y^{j}}{\partial x^{r}}=0 .
\end{gathered}
$$

We want to lift the manifold $v^{\prime r}$ defined by $x^{r+1}=\cdots=x^{p}=0$ $x^{\lambda}=H^{\lambda}\left(x^{1}, \cdots, x^{r}, 0, \cdots, 0\right)$ to an integral manifold of $\Sigma$. Observe that the functions $H^{\lambda}\left(x^{1}, \cdots, x^{r}, 0, \cdots, 0\right)$ are solutions of equations (4). Assume that the equations of $v^{r-1}$ are $x^{r}=\cdots=x^{p}=0$, $x^{\lambda}=H^{\lambda}\left(x^{1}, \cdots, x^{r-1}, 0, \cdots, 0\right), y^{j}=G^{j}\left(x^{1}, \cdots, x^{r-1}\right)$. Equations (5) can be solved with respect to some of the variables $\partial y^{j} / \partial x^{r}$; assume that they can be solved with respect to $\partial y^{1} / \partial x^{r}, \cdots, \partial y^{s} / \partial x^{r}$. Put in (5),

$$
\begin{aligned}
& \frac{\partial x^{r+1}}{\partial x^{r}}=\cdots=\frac{\partial x^{p}}{\partial x^{r}}=0, \quad \frac{\partial x^{\lambda}}{\partial x^{r}}=\frac{\partial H^{\lambda}\left(x^{1}, \cdots, x^{p}\right)}{\partial x^{r}}, \\
& p+1 \leqq \lambda \leqq n^{\prime},
\end{aligned}
$$

and replace the variables $y^{j}$ by arbitrary functions $y^{j}=F^{j}\left(x^{1}, \cdots, x^{r}\right)$, $s+1 \leqq j \leqq n$, subjected to the restrictions $\left(\partial F^{j} / \partial x^{i}\right)_{(0, \cdots, 0)}=0$ and $F^{j}\left(x^{1}, \cdots, x^{r-1}, 0\right)=G^{j}\left(x^{1}, \cdots, x^{r-1}\right), 1 \leqq i \leqq r, s+1 \leqq j \leqq n$. Let $y^{j}=F^{j}\left(x^{1}, \cdots, x^{r}\right), 1 \leqq j \leqq s$, be the solution of the resulting CauchyKowaleswky system with the initial conditions $F^{j}\left(x^{1}, \cdots, x^{r-1}, 0\right)$ $=G^{j}\left(x^{1}, \cdots, x^{r-1}\right), 1 \leqq j \leqq s$. Then, as in the Cartan-Kähler theorem, the manifold $v^{r}$ defined by the equations $x^{r+1}=\cdots=x^{p}=0$, $x^{\lambda}=H^{\lambda}\left(x^{1}, \cdots, x^{r}\right), y^{j}=F^{j}\left(x^{1}, \cdots, x^{r}\right), 1 \leqq j \leqq n$, is a solution of $\Sigma$ which covers $\mathcal{V}^{\prime}$. The Theorem is proved.

\section{REFERENCES}

1. M. Kuranishi, On E. Cartan's prolongation theorem of exterior differential systems, Amer. J. Math. 79 (1957), 1-47.

2. - Lectures on exterior differential systems, Tata Institute of Fundamental Research, Bombay, 1962.

3. M. Kuranishi and A. M. Rodrigues, Quotients of pseudogroups by invariant fiberings, Nagoya J. Math. 24 (1964), 109-128.

Instituto de Pesquisas Matemáticas, Universidade de São Paulo 